\title{
Clinicopathological and functional implications of the inhibitor of apoptosis proteins survivin and XIAP in esophageal cancer
}

\author{
LEVENT DIZDAR ${ }^{1}$, LISA M. JÜNEMANN ${ }^{1}$, THOMAS A. WERNER ${ }^{1}$, PABLO E. VERDE ${ }^{2}$, \\ STEPHAN E. BALDUS ${ }^{3}$, NIKOLAS H. STOECKLEIN ${ }^{1}$, WOLFRAM T. KNOEFEL ${ }^{1}$ and ANDREAS KRIEG ${ }^{1}$ \\ ${ }^{1}$ Department of Surgery (A); ${ }^{2}$ Coordination Centre for Clinical Trials, Heinrich-Heine-University \\ and University Hospital Düsseldorf, D-40225 Düsseldorf; ${ }^{3}$ Institute of Pathology, \\ Cytology and Molecular Pathology, D-51465 Bergisch Gladbach, Germany
}

Received July 4, 2017; Accepted November 21, 2017

DOI: $10.3892 / 01.2018 .7755$

\begin{abstract}
Based on their overexpression and important roles in progression and therapy-resistance in malignant diseases, the inhibitor of apoptosis protein family (IAP) members, survivin and X-linked inhibitor of apoptosis protein (XIAP), represent attractive candidates for targeted therapy. The present study investigated the prognostic and biological relevance of survivin and XIAP in esophageal squamous-cell carcinoma (ESCC) and esophageal adenocarcinoma (EAC). Survivin and XIAP expression was analyzed by immunohistochemistry using tissue microarrays containing 120 ESCC and 90 EAC samples as well as the corresponding non-neoplastic esophageal mucosa samples. IAP expression levels were then correlated to clinicopathological parameters and overall survival to identify any associations. In addition, esophageal cancer cell lines were treated with the survivin inhibitor YM155, and the XIAP inhibitors Birinapant and GDC-0152 in vitro. Survivin and XIAP expression were significantly increased in EAC and ESCC when compared with tumor-adjacent mucosa. In patients with ESCC XIAP expression was associated with female gender and advanced tumor stages, and nuclear survivin expression was associated with poor grading. High XIAP expression was identified as an independent negative prognostic marker in ESCC. By contrast, XIAP inhibitors did not affect cancer cell viability in vitro, and the small molecule survivin inhibitor YM155 significantly reduced cell viability and proliferation in esophageal cancer cell lines. Western blot analysis revealed a dose dependent decrease of survivin accompanied by an increased poly (adenosine diphosphate-ribose) polymerase cleavage following
\end{abstract}

Correspondence to: Professor Andreas Krieg, Department of Surgery (A), Heinrich-Heine-University and University Hospital Düsseldorf, Moorenstr. 5, Building 12.46, D-40225 Düsseldorf, Germany

E-mail: andreas.krieg@med.uni-duesseldorf.de

Key words: esophageal cancer, adenocarcinoma, squamous-cell carcinoma, survivin, XIAP, YM155
YM155 treatment. These findings underline the potential role of survivin and XIAP in the oncogenesis of esophageal cancer and provide a rationale for future clinical studies investigating the therapeutic efficacy of IAP directed therapies in patients with esophageal cancer.

\section{Introduction}

Esophageal cancer is the eighth most common type of cancer worldwide and the sixth leading cause of cancer-related mortality (1). Squamous-cell carcinoma is the predominant histological type, however in the USA, and Western European countries the incidence of esophageal adenocarcinoma (EAC) is steadily rising and exceeds that of esophageal squamous-cell carcinoma (ESCC) (2-6). State-of-the-art treatment algorithms for esophageal cancer consist of multidisciplinary approaches, including surgical resection, combinatory chemo- and radiotherapy, as well as endoscopic procedures $(7,8)$. However, despite aggressive and multimodal treatment concepts the prognosis of patients with esophageal cancer remains disappointing, with overall 5-year survival rates of approximately $20 \%$ (9). Poor outcome in esophageal cancer patients is particularly related to late diagnosis at advanced stages of the disease and high rates of cancer recurrence even after an adequate initial therapy with curative intend (9-11). Unfortunately, the efficacy of current chemo- and radiotherapy regimens has been largely exhausted and further intensification is predominantly associated with an increase in undesirable systemic toxicity. To overcome the difficulty of adverse effects, novel therapeutic concepts focus on the development of targeted anticancer therapies that specifically inhibit aberrant molecular pathways triggered by genomic and proteomic alterations in cancer cells.

In this context, during the last decades the Inhibitor of apoptosis protein (IAP) family attracted considerable attention. Considering their overexpression as well as their association with tumor progression, treatment resistance and poor prognosis in various human cancers, IAPs represent promising targets for cancer therapy. Initially, these proteins were found to function as endogenous inhibitors of caspases, however today it has become increasingly clear that IAPs affect additional cellular functions such as proliferation, migration, invasion and metastasis (12-14). The two most extensively studied members 
of the IAP family are survivin/BIRC5 and X-linked inhibitor of apoptosis protein (XIAP)/BIRC4 (12,14-19). Interestingly, survivin and XIAP have been demonstrated to be important partners accomplishing their antiapoptotic and pro-metastatic functions by direct interaction $(13,20)$.

Aim of this study was to analyze the expression of survivin and XIAP in a large number of tissue specimens from esophageal cancer patients, including primary tumors and tumor adjacent non-malignant mucosa. Expression levels of both IAPs were correlated with clinicopathological variables and overall survival according to the REporting recommendations for tumor MARKer prognostic studies (REMARK) (21). In addition, we analyzed the antitumor activity of small molecule survivin inhibitor YM155 and XIAP inhibitors Birinapant and GDC-0152 in esophageal cancer cell lines originating from both, ESCC as well as EAC.

\section{Materials and methods}

Patient selection and clinicopathological data. Previously constructed tissue microarrays (TMA) containing tissue samples retrieved from human EAC and ESCC were used to assess survivin and XIAP expression (22). All formalin-fixed and paraffin-embedded (FFPE) tissue specimens originated from the Institutes of Pathology of the University Hospitals in Duesseldorf and Cologne. The patients who had undergone radical en bloc esophagectomy and lymphadenectomy with curative intent irrespective of tumor stage and microscopic resection margin at the University Hospital of Duesseldorf and Cologne between 1986 and 2005 were included in this study. Exclusion criteria were preoperative neoadjuvant therapy, macroscopic incomplete resection (R2), esophageal tumors other than squamous cell carcinoma or adenocarcinoma and samples with insufficient tumor material. In addition, 73 tissue samples of tumor adjacent, non-malignant esophageal mucosa were analyzed for survivin and XIAP expression. Information on TNM staging (depth of invasion, lymph node and distant metastasis) as well as grading were retrospectively obtained from the original pathological reports. Data regarding overall survival as well as age at the time of surgery and gender were reviewed. The study was carried out in accordance to Good Clinical Practice, the Declaration of Helsinki and an Institutional Review Board (IRB)-approval of the Medical Faculty, Heinrich Heine University Duesseldorf (IRB-no. 3821) was retrieved.

Immunohistochemistry. Two $\mu \mathrm{m}$ thick sections were cut from each TMA block and mounted on superfrost microscope slides. Immunohistochemical staining was performed as recently described $(23,24)$. Two independent investigators (LD and LMJ) blinded to clinicopathological information evaluated the expression of survivin and XIAP using the immunoreactivity score (IRS) according to Remmele (25). This score is calculated by multiplying the intensity of staining ( 0 , no staining; 1, weak staining; 2 , strong staining; 3 , very strong staining) with the percentage of positive cells ( 0 , no positive cells; 1 , $<10 \%$ positive cells; $2,11-50 \%$ positive cells; $3,51-80 \%$ positive cells; $4,81-100 \%$ positive cells). In case of differing results the samples in question were re-examined by both observers simultaneously and a consensus decision was made.
For survivin, nuclear and cytoplasmic protein expression were separately determined. A tissue slide of pretested human colon and renal cell carcinoma, known to express survivin or XIAP intensively, served as a positive control. Sections incubated with isotype control antibodies were used as negative controls.

Cell lines. ESCC cell lines KYSE30, KYSE270, KYSE410 and KYSE520, established by Shimada et al (26), were obtained from the German collective of microorganisms and cell cultures (DSMZ, Braunschweig, Germany). EAC cell lines OE19 and OE33 were acquired from the European collection of cell cultures (ECACC, Salisbury, UK). All cell lines were maintained in RPMI medium supplemented with $10 \%$ heat inactivated FCS, penicillin and streptomycin at $37^{\circ} \mathrm{C}$ in an atmosphere with $5 \% \mathrm{CO}_{2}$. DNA fingerprinting, conducted as previously described, confirmed that no cross contamination had occurred (27).

Functional in vitro assays. Cell viability and proliferation were assessed in 96-well culture plates with $2 \times 10^{3}$ cells per well. After $24 \mathrm{~h}$ cells were incubated with YM155, Birinapant, GDC-0152 or dimethyl sulfoxid (DMSO) vehicle control for 48 h. The CellTiter $96{ }^{\circledR}$ AQueous Non-Radioactive Cell Proliferation Assay (Promega Corporation,, Madison, WI, USA) was used to measure cell viability. Changes in cell proliferation were quantified based on BrdU-incorporation using a Cell Proliferation ELISA BrdU assay (Roche Applied Science, Mannheim, Germany). Both assays were conducted according to the manufacturer's protocols. Absorbance was measured using the Infinite ${ }^{\circledR} 200$ microplate reader (Tecan Group Ltd., Crailsheim, Germany). The absorbance values of treated cells are presented as a percentage of the absorbance of DMSO treated control cells.

Western blot analysis. $1 \times 10^{5}$ cells were seeded in $25 \mathrm{~cm}^{2}$ cell culture flasks, grown overnight and treated with YM155 or DMSO vehicle control for $24 \mathrm{~h}$. Subsequently, cells were lysed in RIPA buffer (Sigma-Aldrich; Merck KGaA, Darmstadt, Germany) and supplemented with protease inhibitor mix (cOmplete; Roche Diagnostics, Indianapolis, IN, USA). Lysates $(20 \mu \mathrm{g})$ were separated on SDS-PAGE gels and transferred to nitrocellulose membranes. Membranes were blocked with TBS-T buffer containing 5\% nonfat dry milk and incubated with primary antibodies overnight at $4^{\circ} \mathrm{C}$. Blots were washed and incubated with secondary antibodies. Immune-Star ${ }^{\mathrm{TM}}$ Western $C^{\text {тм }}$ Kit (Bio-Rad Laboratories, Inc., Hercules, CA, USA) and the Versa Doc Imaging System (Bio-Rad Laboratories $\mathrm{GmbH}$, Munich, Germany) were used for signal detection. The experiments were repeated three times and one representative western blot (WB) was chosen for presentation.

Reagents. Sepantronium Bromide (YM155), Birinapant and GDC-0152 were purchased from Selleckchem (Houston, TX, USA). Antibodies used for immunohistochemistry (IHC) or WB analysis were raised against survivin (NB500-201; 1:750 dilution for IHC and 1:1,000 dilution for WB; Novus, Littleton, CO, USA), XIAP (clone 48, 1:35 dilution for IHC or Clone 28, 1:1,000 dilution for WB; both BD Biosciences, San Jose, CA, USA), PARP (9542; 1:1,000 dilution; Cell Signaling, Denver, MA, USA), $\alpha$-tubulin (Clone DM1A; 1:5,000 dilution; 
Sigma-Aldrich; Merck KGaA) and GAPDH (Clone 6C5; 1:5,000 dilution; Abcam, Cambridge, UK). Isotype control was performed using mouse IgG1k (MOPC-21; 1:70 dilution; Abcam) and rabbit immunoglobulin fraction (Code X0903; 1:15,000 dilution; Dako, Glostrup, Denmark).

Statistical analysis. Differences of IAP expression levels in esophageal cancer specimens and adjacent non-neoplastic mucosa were analyzed using the Mann-Whitney U test. For numerical data, a correlation between clinicopathological variables and expression levels of survivin or XIAP was examined using the Mann-Whitney U test. The chi-square test was implemented for categorical data. Spearman's correlation coefficient was used to test a relationship between survivin and XIAP expression levels. For some analyses immunoreactivity scores were categorized into high (IRS $>2$ ) or low (IRS $\leq 2$ ) expression of survivin and XIAP, respectively. The cut-off value for this categorization was set according to the median IRS for survivin and XIAP expression in all investigated EC tissue samples. Outcome measures included overall survival, defined as the period from the date of surgery until the date of last follow up or until death of any cause. Patients with incomplete tumor resection or who died within 30 days after operation were excluded from the survival analysis. Kaplan-Meier curves were generated and assessed using the log-rank (Mantel Cox) test and hazard ratios (HRs) with 95\% confidence intervals (CIs) were estimated. For multivariate survival analysis all variables were included into a logistic regression analysis. Analyses were performed using GraphPad Prism for Windows (version 5; GraphPad Software, Inc., La Jolla, CA, USA) and SPSS statistics for Windows (version 17.0; SPSS, Inc., Chicago, IL, USA). P $<0.05$ was considered to indicate a statistically significant difference.

\section{Results}

Patients and outcome. Using our selection criteria, a total number of $90 \mathrm{EAC}$ and $120 \mathrm{ESCC}$ patients who underwent radical en bloc esophagectomy between 1986 and 2005 could be enrolled into our study. Unfortunately, 10 EAC patients and 6 ESCC patients had to be excluded from our analysis because of insufficient evaluable tumor material after immunohistochemical staining procedure. Clinicopathological characteristics of these remaining $80 \mathrm{EAC}$ and $114 \mathrm{ESCC}$ patients are summarized in Table I. The median age of EAC patients at the time of surgery was 66 years (range, 36-82) and 58 years in the group of ESCC patients (range, 37-83). A total of $67 \mathrm{EAC}$ and $108 \mathrm{ESCC}$ patients met all predefined inclusion criteria for our survival analysis. EAC and ESCC patients had a mean follow-up time of 38.0 month (range, 1-120) and 22.8 month (range, 1-120 month), respectively. A total of 47 EAC patients and 89 ESCC patients died during the follow up period. Mean overall survival of EAC patients was 49.3 month (range, 1-120 month; 95\% CI: 38.4-60.2 month) and 28.3 month for ESCC patients (range, 1-120 month; 95\% CI: 21.8-34.8).

Survivin and XIAP expression in esophageal cancer. As expected, immunohistochemical staining of TMAs showed a cytoplasmic and nuclear expression for survivin, whereas
Table I. Patient characteristics.

\begin{tabular}{|c|c|c|}
\hline & EAC & ESCC \\
\hline Variable & $\begin{array}{c}\text { No. of } \\
\text { patients (\%) }\end{array}$ & $\begin{array}{c}\text { No. of } \\
\text { patients (\%) }\end{array}$ \\
\hline Total & 80 & 114 \\
\hline \multicolumn{3}{|l|}{ Age } \\
\hline Median (range); years & $66(36-82)$ & $59(37-83)$ \\
\hline \multicolumn{3}{|l|}{ Sex } \\
\hline Male & $62(77.5)$ & $84(73.7)$ \\
\hline Female & $18(22.5)$ & $30(26.3)$ \\
\hline \multicolumn{3}{|l|}{ Tumor stage } \\
\hline $\mathrm{T} 1 / 2$ & $44(55.0)$ & $39(34.2)$ \\
\hline $\mathrm{T} 3 / 4$ & $33(41.3)$ & $75(65.8)$ \\
\hline Missing & $3(3.8)$ & $0(0)$ \\
\hline \multicolumn{3}{|l|}{ Lymph node metastasis } \\
\hline No & $26(32.5)$ & $39(34.2)$ \\
\hline $\mathrm{N} 1+$ & $51(63.8)$ & $75(65.8)$ \\
\hline Missing & $3(3.8)$ & $0(0)$ \\
\hline \multicolumn{3}{|l|}{ Distant metastasis } \\
\hline M0 & $71(88.8)$ & $112(98.2)$ \\
\hline M1 & $6(7.5)$ & $2(1.8)$ \\
\hline Missing & $3(3.8)$ & $0(0)$ \\
\hline \multicolumn{3}{|l|}{ Grading } \\
\hline $\mathrm{G} 1 / 2$ & $21(26.3)$ & $66(57.9)$ \\
\hline $\mathrm{G} 3 / 4$ & $53(66.3)$ & $48(42.1)$ \\
\hline Missing & $6(7.5)$ & $0(0)$ \\
\hline \multicolumn{3}{|l|}{ Resection status } \\
\hline R0 & $75(93.8)$ & $114(100)$ \\
\hline $\mathrm{R}+$ & $2(2.5)$ & $0(0)$ \\
\hline Missing & $3(3.8)$ & $0(0)$ \\
\hline
\end{tabular}

EAC, n=80; ESCC, n=114; EAC, esophageal adenocarcinoma; ESCC, esophageal squamous-cell cancer.

XIAP was exclusively localized in the cytoplasm (Fig. 1A). Both IAPs were aberrantly expressed in esophageal cancer tissue, when compared to adjacent non-neoplastic tumor mucosa, with significantly increased expression levels in both ESCC and EAC (Fig. 1B). Interestingly, XIAP and nuclear survivin expression levels were significantly higher in EAC when compared to ESCC (Fig. 1B). Of note, cytoplasmic survivin expression correlated positively with XIAP expression in EAC $\left(r_{s}=0.442 ; P<0.001\right)$ (Fig. 1D). In contrast, we did not detect a correlation between survivin and XIAP expression in ESCC samples.

To further elucidate a correlation between survivin or XIAP expression levels and clinicopathological variables, two statistical approaches were used. First we compared the IRS across groups for each clinicopathological parameter. This approach revealed that high XIAP expression strongly correlated with female gender and advanced tumor stages in ESCC patients. Furthermore, high nuclear survivin expression levels were associated with poorly 

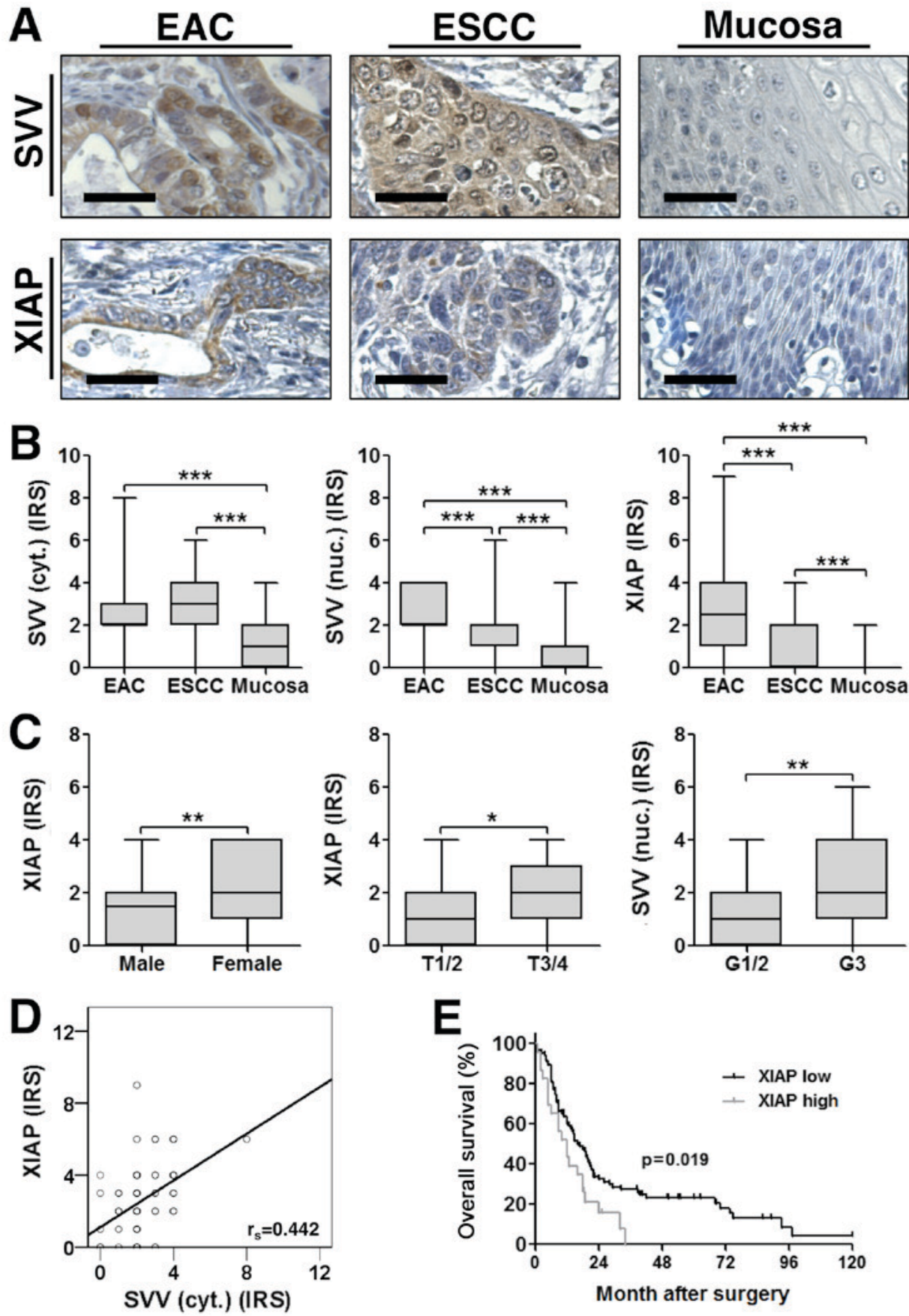

Figure 1. (A) Representative images of immunohistochemical staining for SVV (top) and XIAP (bottom) in EAC, ESCC and tumor adjacent non-malignant mucosa. Images were captured at X400 magnification and scale bars indicate $25 \mu \mathrm{m}$. (B) SVV and XIAP expression were significantly increased in esophageal cancer tissue specimens, when compared to non-malignant mucosa. Furthermore, nuclear SVV and XIAP expression levels were higher in EAC when compared to ESCC (EAC, n=80; ESCC, n=114; NEM, n=73). (C) IAP expression levels and their association with clinicopathological variables. Boxplots display the median IRS with the upper and lower quartile, as well as the maximum and minimum stratified according to the respective clinicopathological variable. [Sex: median IRS female=2 ( $n=30)$; median IRS male=1.5 ( $n=84) ; P=0.003$; T-stage: median IRS T1/2=1 ( $n=39)$; median IRS T3/4=2 ( $=75)$; $P=0.03$; Grading: median IRS G1/2=1 ( $\mathrm{n}=66)$; median IRS G3=2 $(\mathrm{n}=48) ; \mathrm{P}=0.005]$. Data were analyzed using a two-tailed nonparametric Mann-Whitney U test. ${ }^{*} \mathrm{P}<0.05,{ }^{* *} \mathrm{P}<0.01$ and ${ }^{* * *} \mathrm{P}<0.001$, as indicated. (D) XIAP and cytoplasmic SVV expression were positively correlated in corresponding EAC (rs $=0.442$; $\mathrm{P}<0.001$ ). (E) Kaplan-Meier curve represents the prognostic value of XIAP expression in ESCC. SVV, survivin; EAC, esophageal adenocarcinoma; ESCC, esophageal squamous-cell cancer; XIAP, X-linked inhibitor of apoptosis protein; IRS, immunoreactivity score.

differentiated (G3) ESCC (Fig. 1C). In contrast, no significant correlation between survivin or XIAP expression and clinicopathological variables became evident in EAC patients. Next, by categorizing IAP expression into high (IRS $>2$ ) or low (IRS $\leq 2)$ we could confirm the correlation of high XIAP expression and female gender as well as high nuclear survivin expression and poorly differentiated (G3) ESCC (Tables II and III).
XIAP is an independent negative prognostic marker in $E S C C$. Univariate survival analysis of EAC patients, using Kaplan-Meier curves and log-rank test revealed that the presence of lymph node and distant metastases were significantly associated with poor overall survival. However, neither survivin, nor XIAP expression levels correlated with poor prognosis in EAC patients (Table IV). Moreover, multivariate 


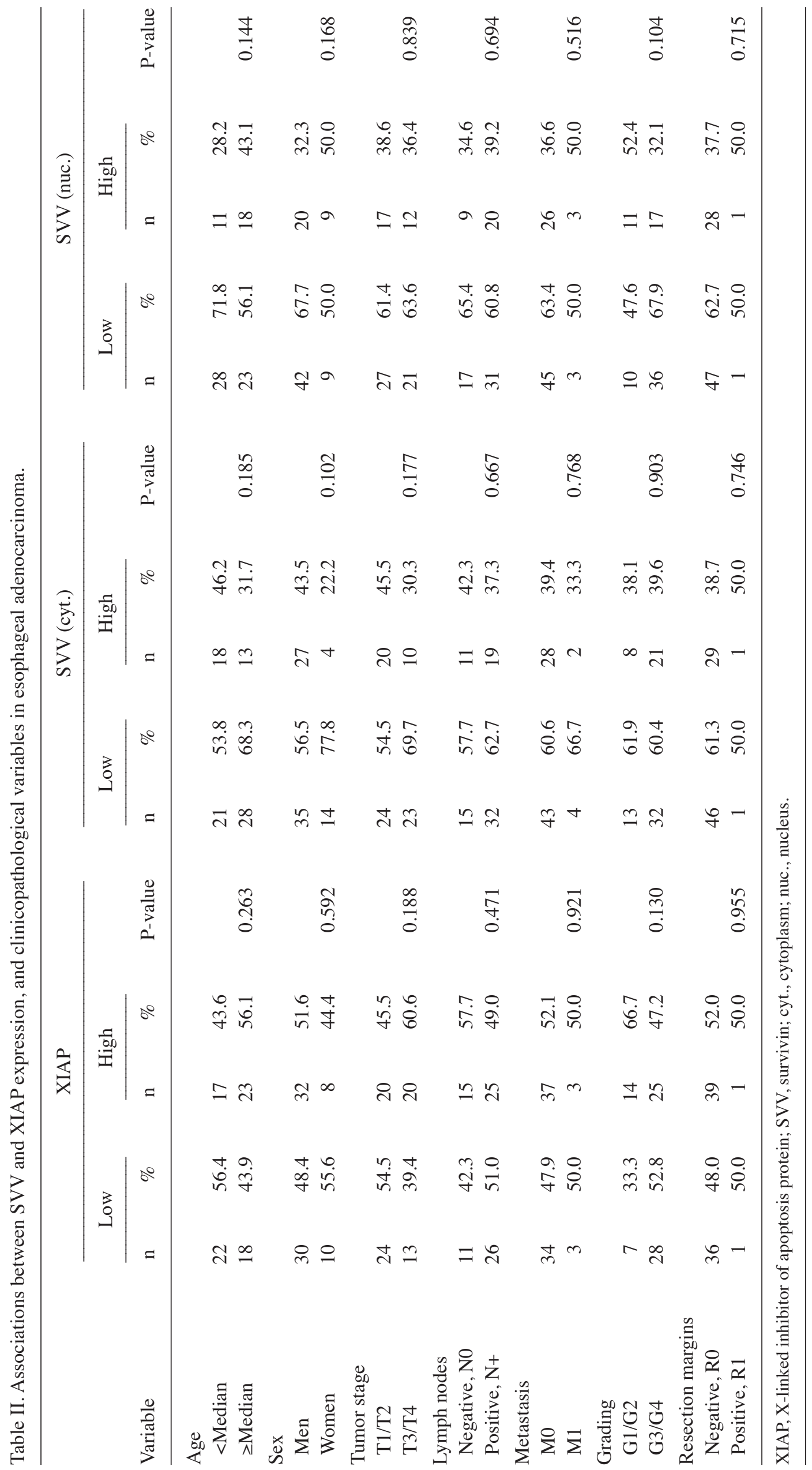




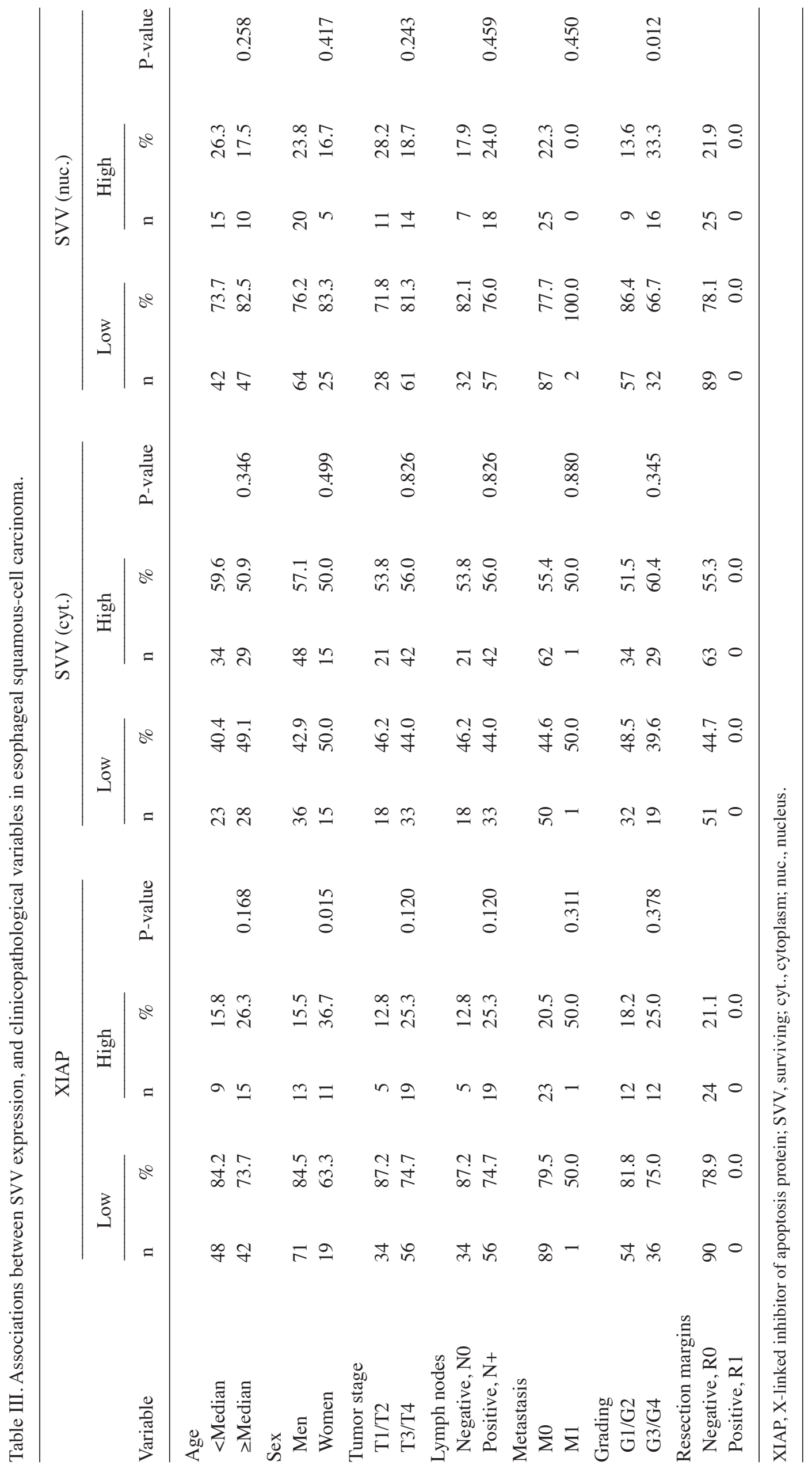


Table IV. Overall survival of esophageal cancer patients: Univariate analysis.

\begin{tabular}{|c|c|c|c|c|c|c|}
\hline \multirow[b]{2}{*}{ Variable } & \multicolumn{3}{|c|}{ Adenocarcinoma } & \multicolumn{3}{|c|}{ Squamous cell carcinoma } \\
\hline & HR & $95 \% \mathrm{CI}$ & P-value & HR & $95 \% \mathrm{CI}$ & P-value \\
\hline Age & 1.369 & $0.756-2.478$ & 0.279 & 1.092 & $0.713-1.671$ & 0.683 \\
\hline Sex & 0.901 & $0.421-1.930$ & 0.781 & 1.328 & $0.819-2.154$ & 0.243 \\
\hline T $1 / 2$ vs. T $3 / 4$ & 1.339 & $0.748-2.399$ & 0.306 & 1.491 & $0.957-2.323$ & 0.072 \\
\hline N0 vs. N+ & 2.173 & $1.097-4.305$ & 0.018 & 1.348 & $0.865-2.102$ & 0.181 \\
\hline M0 vs. M+ & 12.91 & $2.571-64.82$ & $<0.001$ & 3.445 & $0.833-14.240$ & 0.065 \\
\hline G $1 / 2$ vs. G 3/4 & 1.582 & $0.759-3.297$ & 0.199 & 1.285 & 0.843-1.959 & 0.237 \\
\hline XIAP high vs. low & 0.931 & $0.524-1.653$ & 0.798 & 1.798 & $1.087-2.973$ & 0.019 \\
\hline SVV (cyt.) high vs. low & 1.222 & $0.687-2.175$ & 0.478 & 0.870 & $0.571-1.326$ & 0.513 \\
\hline SVV (nuc.) high vs. low & 0.841 & $0.460-1.539$ & 0.559 & 1.066 & $0.651-1.744$ & 0.797 \\
\hline
\end{tabular}

HR, hazard ratio; CI, confidence interval; cyt., cytoplasm; nuc., nucleus; XIAP, X-linked inhibitor of apoptosis protein; SVV, survivin.

Table V. Overall survival of patients with esophageal cancer: Multivariate analysis.

\begin{tabular}{lcccccc}
\hline & \multicolumn{3}{c}{ Adenocarcinoma } & & \multicolumn{2}{c}{ Squamous cell carcinoma } \\
\cline { 2 - 5 } Variable & HR & $95 \%$ CI & P-value & & HR & $95 \%$ CI \\
\hline M0 vs. M+ & 18.264 & $3.290-101.4$ & 0.001 & $/$ & P-value \\
XIAP, high vs. low & $/$ & $/$ & $/$ & 1.798 & $1.087-2.973$ \\
\hline
\end{tabular}

HR, hazard ratio; CI, confidence interval; XIAP, X-linked inhibitor of apoptosis protein.

logistic regression analysis confirmed the presence of distant metastasis as independent negative prognostic factor in EAC patients (Table V).

In contrast to these findings, univariate analysis in the group of ESCC patients revealed that high levels of XIAP expression were significantly associated with a poor prognosis (Fig. 1E; Table IV). Importantly, multivariate logistic regression analysis confirmed high XIAP expression levels as an independent negative prognostic factor in our cohort of ESCC patients (Table V).

In vitro effects of survivin and XIAP directed therapy in esophageal cancer cells. A compilation of cell lines originating from human esophageal cancer comprising four ESCC (KYSE30, KYSE270, KYSE410 and KYSE520) and two EAC (OE19 and OE33) cell lines were analyzed for survivin and XIAP expression using Western blot analysis. As shown in Fig. 2A survivin and XIAP expression were detectable in all esophageal cancer cell lines independent of their histological subtype. To explore the effect of a small molecule mediated inhibition of survivin and XIAP on esophageal cancer cell viability, we incubated ESCC as well as EAC cell lines with increasing concentrations of the survivin antagonist YM155 and the XIAP antagonists Birinapant and GDC-0152. YM155 decreased the cell viability dose dependently in all investigated cell lines, with $\mathrm{IC}_{50}$ values ranging between 4.6 and $23.6 \mathrm{nM}$ (Fig. 2B). In contrast, XIAP inhibitors Birinapant and GDC-0152 exhibited no measurable effect on cancer cell viability (Fig. 2B). As only YM155 demonstrated in vitro cell growth inhibitory effects, we focused our further analysis on this small molecule survivin inhibitor. Comparable to the effect observed on cell viability, YM155 treatment significantly reduced cell proliferation of ESCC and EAC cells as measured by BrdU incorporation (Fig. 2C). Since YM155 has been demonstrated to execute its anti-tumor effects through inhibition of survivin mRNA transcription, we analyzed survivin protein expression in YM155 treated cells using Western blot analysis. As expected, YM155 treatment decreased survivin protein levels in all esophageal cancer cell lines accompanied by a PARP cleavage, indicating apoptotic cell death (Fig. 3). Of note, YM155 also induced a decrease in XIAP expression levels in KYSE270 and KYSE 520 cell lines (Fig. 3).

\section{Discussion}

Given their importance in therapy-resistance and tumor progression, IAP family members survivin and XIAP display promising biomarkers and novel druggable targets for innovative anti-cancer therapies $(14,28)$. Notably, both IAPs act synergistically in many respects, as they stabilize each other and realize their antiapoptotic and pro-metastatic functions by direct interaction $(13,20)$. Published data on survivin and XIAP expression in esophageal cancer are in part controversial or even incomplete and none of the studies 

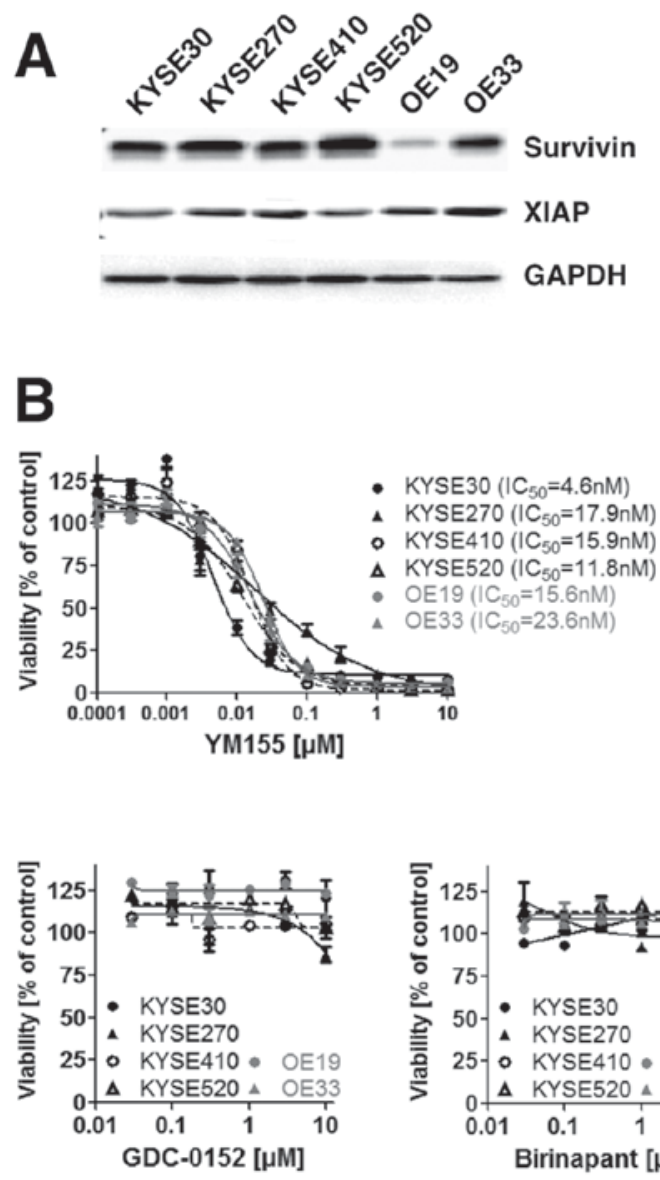
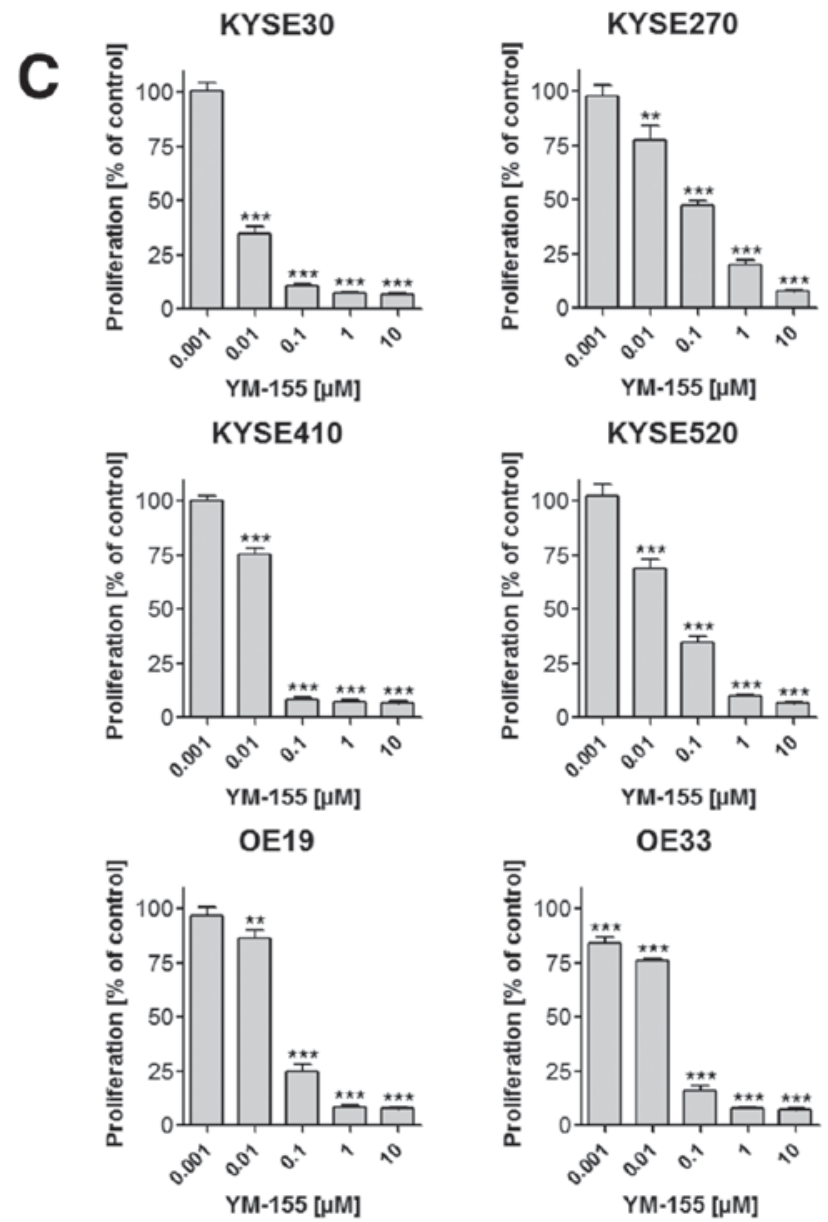

Figure 2. (A) Western blotting confirmed survivin and XIAP expression in all esophageal cancer cell lines. GAPDH served as the loading control. (B) The survivin inhibitor, YM155 induced a dose dependent decrease in cell viability. By contrast, Smac mimetics GDC-0152 and Birinapant had no effect on cancer cell viability (C) YM155 treatment significantly reduced cell proliferation, measured by bromodeoxyuridine incorporation in esophageal adeno- and squamous-cell carcinoma cell lines. DMSO served as the vehicle control. Cell viability or proliferation of treated cells is presented as a percentage of the viability or proliferation of DMSO treated control cells. Assays were performed in triplicate. Statistical significance was calculated by a two-tailed nonparametric Mann-Whitney test. ${ }^{* *} \mathrm{P} \leq 0.01$ and ${ }^{* * *} \mathrm{P} \leq 0.001$ vs. control (DMSO). XIAP, X-linked inhibitor of apoptosis protein; YM155, sepantronium bromide; Smac, second mitochondrial-derived activator of caspases.

reported so far has analyzed survivin and XIAP expression in the same cohort of esophageal cancer patients. In ESCC survivin expression could be correlated with clinicopathological parameters and was linked to poor survival in the majority of published studies (29-36). In contrast, the prognostic impact of XIAP expression and its association to pathological variables in ESCC patients has not yet been adequately investigated. To date only one published study exists, demonstrating a correlation of high XIAP expression and poor survival in a collective of ESCC patients treated with adjuvant radiotherapy after radical esophagectomy (37). Moreover, the value of survivin and XIAP expression in EAC patients is even less clear and available data more limited. To the best of our knowledge, studies investigating XIAP expression in EAC patients have not been published yet and only two studies analyzing survivin expression exist. However, the results of these two studies are contradictory. Whereas, Malhotra et al (38) demonstrated an association between high survivin expression and an increased risk of death, Rosato et al (29) detected no prognostic relevance for survivin expression in their collective of 56 EAC patients. Furthermore, both studies did not comprehensively analyze correlations between survivin expression and clinicopathological parameters.

Consistent with, previous reports we could show that cytoplasmic and nuclear survivin, as well as XIAP expression are significantly increased in EAC and ESCC tissue specimens, when compared to non-malignant tumor adjacent mucosa $(32,38-40)$. This cancer specific expression pattern represents an important basis for the use of both proteins in targeted therapies. Another interesting finding of our analysis was the observation that expression levels of cytoplasmic survivin and XIAP were significantly correlated in EAC specimens, indicating the important role of their intermolecular cooperation. Interestingly, Dohi et al $(20,41)$ could demonstrate that survivin and XIAP interaction predominantly takes place inside the mitochondria and cytoplasm. Particularly non-phosphorylated, mitochondrial survivin stabilizes XIAP and protects it from polyubiquitination and subsequent proteosomal degradation $(20,41)$.

Whereas in EAC patients a correlation between survivin or XIAP expression levels and clinicopathological parameters became not evident, in ESCC high XIAP expression was associated with female gender and advanced tumor stages. 


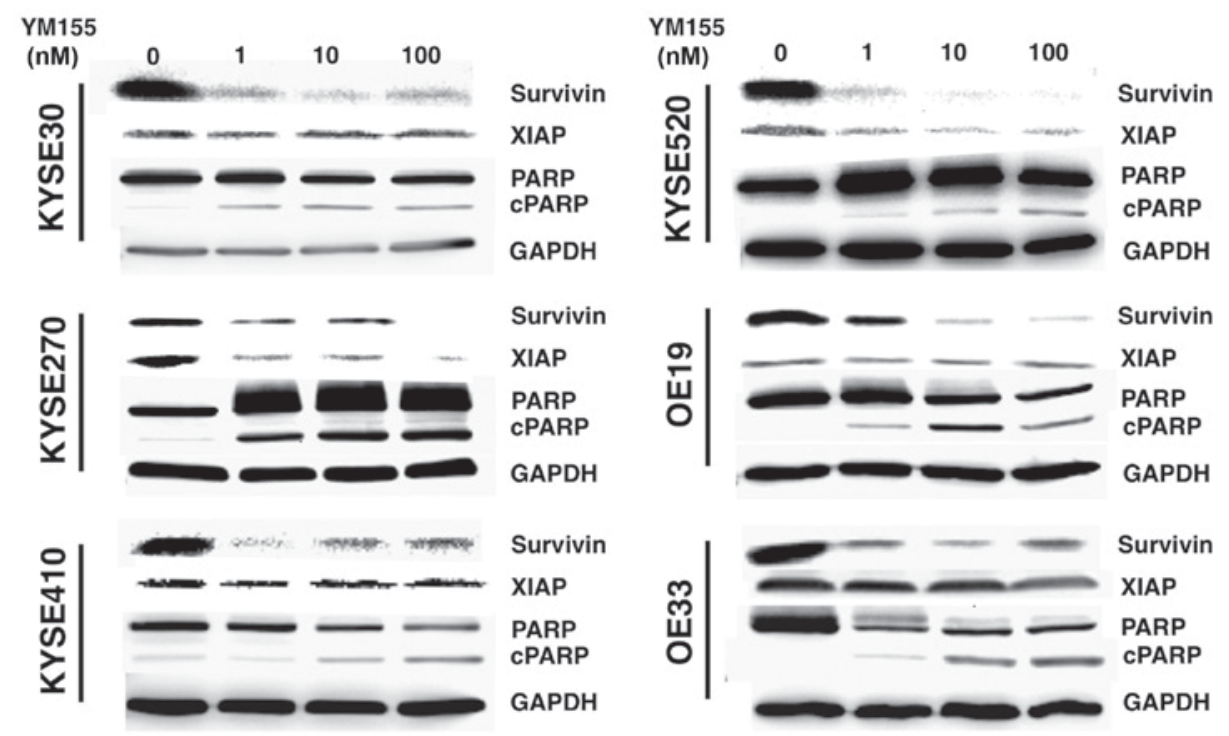

Figure 3. Western blot analysis revealed a reduced survivin expression, as well as PARP cleavage following YM155 treatment for $24 \mathrm{~h}$ in all investigated cell lines. Furthermore, YM155 induced a dose dependent decrease in XIAP expression in the cell lines KYSE270 and KYSE520. Lysates were separated by SDS-PAGE and immunoblotted for the proteins indicated. GAPDH served as loading control. PARP, poly (adenosine diphosphate-ribose) polymerase; cPARP, cleaved PARP; YM155, sepantronium bromide; XIAP, X-linked inhibitor of apoptosis protein.

In addition, nuclear survivin was linked to poorly differentiated (G3) ESCC. Consistent with our findings, Zhou et al (37) detected high XIAP expression levels in advanced ESCC and Takeno et al (30) revealed that increased survivin expression levels were associated with poorly differentiated esophageal cancer. Thus, suggesting a potential role of both IAPs in ESCC progression.

Although a prognostic value of survivin expression in esophageal cancer has been reported by several studies (29-33,36,38), there exist some conflicting data $(29,42,43)$. In this context, our analysis revealed no significant correlation between survivin expression and overall survival in ESCC or EAC patients. In contrast to survivin, much less effort has been spent in investigating the prognostic value of XIAP expression in esophageal cancer. To date, only Zhou et al (37) reported that high levels of XIAP expression were associated with poor outcome in ESCC patients. Of note, our analysis verified the prognostic relevance of XIAP in ESCC patients. However, for EAC patients a prognostic relevance of XIAP became not evident.

To elucidate the potential of an IAP directed therapy in esophageal cancer we made use of the most advanced small-molecule survivin inhibitor YM155, known to repress survivin promoter activity by disrupting proteins like SP1 and interleukin enhancer-binding factor-3 $(44,45)$. In this context, Qin et al (46) and Zhao et al (47) published promising results concerning the antitumor effects of YM155 treatment in ESCC cell lines. However, to the best of our knowledge, for the first time we report also effects of YM155 in EAC. We could show that the small molecule survivin inhibitor effectively reduces survivin expression, cell viability and proliferation in esophageal cancer cell lines, irrespective of their histologic subtype. In addition, we detected considerable PARP cleavage in all investigated EAC and ESCC cell lines after YM155 treatment, indicating apoptotic cell death (48). Consistent with our results,
Qin et al (46) demonstrated decreased cell viability and survivin expression as well as a significant PARP cleavage in YM155 treated ESCC cells. Moreover, they reported that the small-molecule survivin inhibitor enhanced radiosensitization by the abrogation of $\mathrm{G}_{2}$ checkpoint and the inhibition of homologous recombination repair (46). In contrast to our findings, Zhao et al (47) observed that YM155 did not trigger apoptosis, but induced parthanatos, a cell death depending on PARP-1 hyper-activation in ESCC cell lines KYSE410 and KYSE150 (47). However, the key message of both published studies investigating the effects of YM155 treatment in esophageal cancer so far is that YM155 kills esophageal cancer cells and represents a promising tool for novel therapeutic approaches in esophageal cancer patients.

In addition, we tested the effects of the small molecule XIAP antagonists Birinapant and GDC-0152. Both compounds mimic the effects of the second mitochondrial-derived activator of caspases (Smac), which acts as an endogenous antagonist of XIAP, cIAP1 and cIAP2 (49-51). In contrast to YM155, both small molecule IAP antagonists failed to achieve cytotoxic effects on esophageal cancer cells in vitro. Although smac mimetics have been shown to promote cell death by competing with caspases for binding to the BIR domains of XIAP, cIAP1 or cIAP2 (52-54), these IAP-antagonizing compounds turned out to exhibit single agent activity only in a small subset of tumor cells $(54,55)$. This observation might be explained by different mechanisms contributing to Smac mimetic resistance in malignant cells including a tumor necrosis factor $\alpha(\mathrm{TNF} \alpha)$ mediated up-regulation of cIAP2, the inability to form a ripoptosome complex or a defective PI3K signaling pathway $(56,57)$.

In conclusion, our analysis of survivin and XIAP protein expression in esophageal cancer tissue specimens revealed that only XIAP may be regarded as a prognostic marker in ESCC but not in EAC. In addition, small-molecule survivin inhibitor YM155 induced impressive cytotoxic effects on 
esophageal cancer cells in vitro even at nanomolar concentrations. Unfortunately small molecule XIAP antagonists did not exhibit single agent activity in our experiments. However, as they might be effective in combination therapies with other chemotherapeutics or radiation, we suggest that future studies should investigate the efficacy of Smac mimetic-based combination therapies in esophageal cancer.

Despite the limitations of the study including its retrospective design and the lack of in vivo experiments, our findings underline the potential role of survivin and XIAP in the oncogenesis of esophageal cancer and provide a rationale for future clinical studies investigating the therapeutic efficacy of IAP directed therapies in esophageal cancer patients.

\section{References}

1. Ferlay J, Soerjomataram I, Ervik M, Dikshit R, Eser S, Mathers C Rebelo M, Parkin DM, Forman D and Bray F: GLOBOCAN 2012 v1.0, Cancer Incidence and Mortality Worldwide: IARC CancerBase No. 11 (Internet). International Agency for Research on Cancer, Lyon, 2013. http://globocan.iarc.fr. Accessed February 28, 2017.

2. Lepage C, Rachet B, Jooste V, Faivre J and Coleman MP: Continuing rapid increase in esophageal adenocarcinoma in England and Wales. Am J Gastroenterol 103: 2694-2699, 2008.

3. Pohl H and Welch HG: The role of overdiagnosis and reclassification in the marked increase of esophageal adenocarcinoma incidence. J Natl Cancer Inst 97: 142-146, 2005.

4. Eslick GD: Epidemiology of esophageal cancer. Gastroenterol Clin North Am 38: 17-25, vii, 2009.

5. Crane LM, Schaapveld M, Visser O, Louwman MW, Plukker JT and van Dam GM: Oesophageal cancer in The Netherlands: Increasing incidence and mortality but improving survival. Eur J Cancer 43: 1445-1451, 2007.

6. Stavrou EP, McElroy HJ, Baker DF, Smith G and Bishop JF: Adenocarcinoma of the oesophagus: Incidence and survival rates in New South Wales, 1972-2005. Med J Aust 191: 310-314, 2009.

7. Le Bras GF, Farooq MH, Falk GW and Andl CD: Esophageal cancer: The latest on chemoprevention and state of the art therapies. Pharmacol Res 113: 236-244, 2016.

8. Pennathur A, Gibson MK, Jobe BA and Luketich JD: Oesophageal carcinoma. Lancet 381: 400-412, 2013.

9. Howlader N, Noone AM, Krapcho M, Miller D, Bishop K, Altekruse SF, Kosary CL, Yu M, Ruhl J, Tatalovich Z, et al: SEER cancer statistics review, 1975-2013. National Cancer Institute, Bethesda, MD, 2016. http://seercancergov/csr/1975_2013/. Accessed February 28, 2017.

10. Mariette C, Balon JM, Piessen G, Fabre S, Van Seuningen I and Triboulet JP: Pattern of recurrence following complete resection of esophageal carcinoma and factors predictive of recurrent disease. Cancer 97: 1616-1623, 2003.

11. Nakagawa S, Kanda T, Kosugi S, Ohashi M, Suzuki T and Hatakeyama K: Recurrence pattern of squamous cell carcinoma of the thoracic esophagus after extended radical esophagectomy with three-field lymphadenectomy. J Am Coll Surg 198: 205-211, 2004.

12. Mita AC, Mita MM, Nawrocki ST and Giles FJ: Survivin: Key regulator of mitosis and apoptosis and novel target for cancer therapeutics. Clin Cancer Res 14: 5000-5005, 2008.

13. Mehrotra S, Languino LR, Raskett CM, Mercurio AM, Dohi T and Altieri DC: IAP regulation of metastasis. Cancer Cell 17: 53-64, 2010.

14. Fulda S and Vucic D: Targeting IAP proteins for therapeutic intervention in cancer. Nat Rev Drug Discov 11: 109-124, 2012.

15. Altieri DC: New wirings in the survivin networks. Oncogene 27: 6276-6284, 2008

16. Altieri DC: Survivin-The inconvenient IAP. Semin Cell Dev Biol 39: 91-96, 2015

17. Dohi T, Beltrami E, Wall NR, Plescia J and Altieri DC: Mitochondrial survivin inhibits apoptosis and promotes tumorigenesis. J Clin Invest 114: 1117-1127, 2004.

18. Reed JC and Bischoff JR: BIRinging chromosomes through cell division-and survivin' the experience. Cell 102: 545-548, 2000.
19. Fulda S: Regulation of cell migration, invasion and metastasis by IAP proteins and their antagonists. Oncogene 33: 671-676, 2014.

20. Dohi T, Okada K, Xia F, Wilford CE, Samuel T, Welsh K, Marusawa $\mathrm{H}$, Zou H, Armstrong R, Matsuzawa S, et al: An IAP-IAP complex inhibits apoptosis. J Biol Chem 279: 34087-34090, 2004.

21. McShane LM, Altman DG, Sauerbrei W, Taube SE, Gion M and Clark GM; Statistics Subcommittee of the NCI-EORTC Working Group on Cancer Diagnostics: REporting recommendations for tumour MARKer prognostic studies (REMARK). Br J Cancer 93: 387-391, 2005.

22. Schmelzle M, Dizdar L, Matthaei H, Baldus SE, Wolters J, Lindenlauf N, Bruns I, Cadeddu RP, Kröpil F, Topp SA, et al: Esophageal cancer proliferation is mediated by cytochrome P450 2C9 (CYP2C9). Prostaglandins Other Lipid Mediat 94: 25-33, 2011

23. Dizdar L, Oesterwind KA, Riemer JC, Werner TA, Mersch S, Möhlendick B, Schutte SC, Verde PE, Raba K, Topp SA, et al: Preclinical assesement of survivin and XIAP as prognostic biomarkers and therapeutic targets in gastroenteropancreatic neuroendocrine neoplasia. Oncotarget 8: 8369-8382, 2017.

24. Werner TA, Tamkan-Ölcek Y, Dizdar L, Riemer JC, Wolf A, Cupisti K, Verde PE, Knoefel WT and Krieg A: Survivin and XIAP: Two valuable biomarkers in medullary thyroid carcinoma. Br J Cancer 114: 427-434, 2016.

25. Remmele W and Stegner HE: Recommendation for uniform definition of an immunoreactive score (IRS) for immunohistochemical estrogen receptor detection (ER-ICA) in breast cancer tissue. Pathologe 8: 138-140, 1987 (In German).

26. Shimada $\mathrm{Y}$, Imamura $\mathrm{M}$, Wagata $\mathrm{T}$, Yamaguchi $\mathrm{N}$ and Tobe $\mathrm{T}$ : Characterization of 21 newly established esophageal cancer cell lines. Cancer 69: 277-284, 1992.

27. Krieg A, Mersch S, Boeck I, Dizdar L, Weihe E, Hilal Z, Krausch M, Möhlendick B, Topp SA, Piekorz RP, et al: New model for gastroenteropancreatic large-cell neuroendocrine carcinoma: Establishment of two clinically relevant cell lines. PLoS One 9: e88713, 2014

28. Tamm I, Richter S, Oltersdorf D, Creutzig U, Harbott J, Scholz F, Karawajew L, Ludwig WD and Wuchter C: High expression levels of $x$-linked inhibitor of apoptosis protein and survivin correlate with poor overall survival in childhood de novo acute myeloid leukemia. Clin Cancer Res 10: 3737-3744, 2004.

29. Rosato A, Pivetta M, Parenti A, Iaderosa GA, Zoso A, Milan G, Mandruzzato S, Del Bianco P, Ruol A, Zaninotto G and Zanovello P: Survivin in esophageal cancer: An accurate prognostic marker for squamous cell carcinoma but not adenocarcinoma. Int J Cancer 119: 1717-1722, 2006.

30. Takeno S, Yamashita S, Takahashi Y, Ono K, Kamei M, Moroga T and Kawahara K: Survivin expression in oesophageal squamous cell carcinoma: Its prognostic impact and splice variant expression. Eur J Cardiothorac Surg 37: 440-445, 2010.

31. Xia H, Chen S, Huang H and Ma H: Survivin over-expression is correlated with a poor prognosis in esophageal cancer patients. Clin Chim Acta 446: 82-85, 2015.

32. Grabowski P, Kühnel T, Mühr-Wilkenshoff F, Heine B, Stein H, Höpfner M, Germer CT and Scherübl H: Prognostic value of nuclear survivin expression in oesophageal squamous cell carcinoma. Br J Cancer 88: 115-119, 2003.

33. Mega S, Miyamoto M, Li L, Kadoya M, Takahashi R, Hase R, Kaneko H, Shichinohe T, Kawarada Y, Itoh T, et al: Immunohistochemical analysis of nuclear survivin expression in esophageal squamous cell carcinoma. Dis Esophagus 19: $355-359,2006$

34. Zhu H, Wang Q, Hu C, Zhang W, Quan L, Liu M, Xu N and Xiao Z: High expression of survivin predicts poor prognosis in esophageal squamous cell carcinoma following radiotherapy. Tumour Biol 32: 1147-1153, 2011.

35. Hui MK, Lai KK, Chan KW, Luk JM, Lee NP, Chung Y, Cheung LC, Srivastava G, Tsao SW, Tang JC and Law S: Clinical correlation of nuclear survivin in esophageal squamous cell carcinoma. Med Oncol 29: 3009-3016, 2012.

36. Hsu KF, Lin CK, Yu CP, Tzao C, Lee SC, Lee YY, Tsai WC and Jin JS: Cortactin, fascin, and survivin expression associated with clinicopathological parameters in esophageal squamous cell carcinoma. Dis Esophagus 22: 402-408, 2009.

37. Zhou S, Ye W, Shao Q, Qi Y, Zhang M and Liang J: Prognostic significance of XIAP and NF- $\mathrm{B}$ expression in esophageal carcinoma with postoperative radiotherapy. World J Surg Oncol 11: $288,2013$. 
38. Malhotra U, Zaidi AH, Kosovec JE, Kasi PM, Komatsu Y, Rotoloni CL, Davison JM, R C, Irvin, Hoppo T, et al: Prognostic value and targeted inhibition of survivin expression in esophageal adenocarcinoma and cancer-adjacent squamous epithelium. PLoS One 8: e78343, 2013.

39. Zhang S, Ding F, Luo A, Chen A, Yu Z, Ren S, Liu Z and Zhang L: XIAP is highly expressed in esophageal cancer and its downregulation by RNAi sensitizes esophageal carcinoma cell lines to chemotherapeutics. Cancer Biol Ther 6: 973-980, 2007.

40. Nemoto T, Kitagawa M, Hasegawa M, Ikeda S, Akashi T, Takizawa T, Hirokawa K and Koike M: Expression of IAP family proteins in esophageal cancer. Exp Mol Pathol 76: 253-259, 2004.

41. Dohi T, Xia F and Altieri DC: Compartmentalized phosphorylation of IAP by protein kinase A regulates cytoprotection. Mol Cell 27: 17-28, 2007.

42. Dabrowski A, Filip A, Zgodziński W, Dabrowska M, Polańska D, Wójcik M, Zinkiewicz K and Wallner G: Assessment of prognostic significance of cytoplasmic survivin expression in advanced oesophageal cancer. Folia Histochem Cytobiol 42: 169-172, 2004.

43. Warnecke-Eberz U, Hokita S, Xi H, Higashi H, Baldus SE, Metzger R, Brabender J, Bollschweiler E, Mueller RP, Dienes HP, et al: Overexpression of survivin mRNA is associated with a favorable prognosis following neoadjuvant radiochemotherapy in esophageal cancer. Oncol Rep 13: 1241-1246, 2005.

44. Yamauchi T, Nakamura N, Hiramoto M, Yuri M, Yokota $\mathrm{H}$ Naitou M, Takeuchi M, Yamanaka K, Kita A, Nakahara T, et al: Sepantronium bromide (YM155) induces disruption of the ILF3/p54(nrb) complex, which is required for survivin expression. Biochem Biophys Res Commun 425: 711-716, 2012.

45. Cheng Q, Ling X, Haller A, Nakahara T, Yamanaka K, Kita A, Koutoku H, Takeuchi M, Brattain MG and Li F: Suppression of survivin promoter activity by YM155 involves disruption of Spl-DNA interaction in the survivin core promoter. Int $\mathbf{J}$ Biochem Mol Biol 3: 179-197, 2012.

46. Qin Q, Cheng H, Lu J, Zhan L, Zheng J, Cai J, Yang X, Xu L, Zhu H, Zhang C, et al: Small-molecule survivin inhibitor YM155 enhances radiosensitization in esophageal squamous cell carcinoma by the abrogation of G2 checkpoint and suppression of homologous recombination repair. J Hematol Oncol 7: 62, 2014.
47. Zhao N, Mao Y, Han G, Ju Q, Zhou L, Liu F, Xu Y and Zhao X: YM155, a survivin suppressant, triggers PARP-dependent cell death (parthanatos) and inhibits esophageal squamous-cell carcinoma xenografts in mice. Oncotarget 6: 18445-18459, 2015.

48. Kaufmann SH, Desnoyers S, Ottaviano Y, Davidson NE and Poirier GG: Specific proteolytic cleavage of poly (ADP-ribose) polymerase: An early marker of chemotherapy-induced apoptosis. Cancer Res 53: 3976-3985, 1993.

49. Benetatos CA, Mitsuuchi Y, Burns JM, Neiman EM, Condon SM, Yu G, Seipel ME, Kapoor GS, Laporte MG, Rippin SR, et al: Birinapant (TL32711), a bivalent SMAC mimetic, targets TRAF2-associated cIAPs, abrogates TNF-induced NF- $\kappa$ B activation, and is active in patient-derived xenograft models. Mol Cancer Ther 13: 867-879, 2014.

50. Flygare JA, Beresini M, Budha N, Chan H, Chan IT, Cheeti S, Cohen F, Deshayes K, Doerner K, Eckhardt SG, et al: Discovery of a potent small-molecule antagonist of inhibitor of apoptosis (IAP) proteins and clinical candidate for the treatment of cancer (GDC-0152). J Med Chem 55: 4101-4113, 2012.

51. Du C, Fang M, Li Y, Li L and Wang X: Smac, a mitochondrial protein that promotes cytochrome c-dependent caspase activation by eliminating IAP inhibition. Cell 102: 33-42, 2000.

52. Liu Z, Sun C, Olejniczak ET, Meadows RP, Betz SF, Oost T, Herrmann J, Wu JC and Fesik SW: Structural basis for binding of Smac/DIABLO to the XIAP BIR3 domain. Nature 408: 1004-1008, 2000.

53. Wu G, Chai J, Suber TL, Wu JW, Du C, Wang X and Shi Y: Structural basis of IAP recognition by Smac/DIABLO. Nature 408: 1008-1012, 2000

54. Fulda S: Smac mimetics as IAP antagonists. Semin Cell Dev Biol 39: 132-138, 2015.

55. Bai L, Smith DC and Wang S: Small-molecule SMAC mimetics as new cancer therapeutics. Pharmacol Ther 144: 82-95, 2014.

56. Petersen SL, Peyton M, Minna JD and Wang X: Overcoming cancer cell resistance to Smac mimetic induced apoptosis by modulating cIAP-2 expression. Proc Natl Acad Sci USA 107: 11936-11941, 2010.

57. Maas C, Tromp JM, van Laar J, Thijssen R, Elias JA, Malara A, Krippner-Heidenreich A, Silke J, van Oers MH and Eldering E: CLL cells are resistant to smac mimetics because of an inability to form a ripoptosome complex. Cell Death Dis 4: e782, 2013. 